\title{
Prognostic performance of qSOFA in oncology patients admitted to the emergency department with suspected infection
}

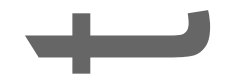

Tze Lui Koh ${ }^{1}$, Emmanuel Canet ${ }^{2}$, Sobia Amjad ${ }^{3}$, Rinaldo Bellomo ${ }^{4}$, David Taylor ${ }^{3,5}$, Hui K Gan $^{6,7,8}$, Nada Marhoon ${ }^{6}$, Andrew Lim ${ }^{6}$, Wee Loon Ong ${ }^{1,9,10,11}$, Vivek Krishnan ${ }^{12}$, Richard Khor $^{1}$

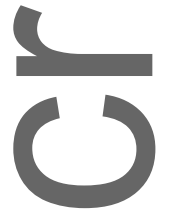

${ }^{1}$ Department of Radiation Oncology, Olivia Newton-John Cancer Wellness and Research

Centre, Austin Health, VIC, Australia

${ }^{2}$ Medical ICU, Hôtel-Dieu, University Hospital, Nantes, Loire-Atlantique, France

${ }^{3}$ University of Melbourne, Parkville, VIC, Australia

${ }^{4} \mathrm{ICU}$, Austin Health, Heidelberg, VIC, Australia

${ }^{5}$ Emergency Department, Austin Health, Heidelberg, VIC, Australia

${ }^{6}$ Medical Oncology, Olivia Newton-John Cancer Wellness and Research Centre, Austin Health, VIC, Australia

${ }^{7}$ La Trobe University School of Cancer Medicine, Heidelberg, VIC, Australia

${ }^{8}$ Department of Medicine, University of Melbourne, Heidelberg, VIC, Australia

${ }^{9}$ Department of Epidemiology and Preventive Medicine, Monash University, Melbourne, VIC,

Australia

${ }^{10}$ Health and Biomedical Informatics Centre, University of Melbourne, Melbourne, VIC, Australia

${ }^{11}$ School of Clinical Medicine, University of Cambridge, Cambridge, UK

${ }^{12}$ MKM Health, South Yarra, VIC, Australia

Short title: qSOFA in cancer patients with infection

This is the author manuscript accepted for publication and has undergone full peer review but has not been through the copyediting, typesetting, pagination and proofreading process, which may lead to differences between this version and the Version of Record. Please cite this article as doi:

10.1111/ajco.13422.

This article is protected by copyright. All rights reserved. 
Abstract: $245 / 250$ words

Word count: $2191 / 3500$ words

Table/s Figures: 3 tables and 2 figures $/ 5$ tables or figures

Number of references: $14 / 50$

Corresponding author:

Dr Tze Lui Koh

Department of Radiation Oncology

Olivia Newton-John Cancer Wellness and Research Centre, Austin Health

145 Studley Road, Heidelberg 3084 VIC

P: 03 9496 2800;

F: 0394962826

E: Violet koh@hótmail.com
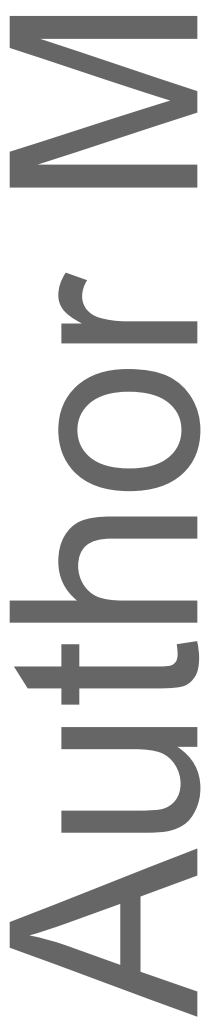

This article is protected by copyright. All rights reserved. 


\section{ABSTRACT}

Aim: We aimed to test the performance of the quick Sequential Organ Failure Assessment score (qSOFA) in predicting the outcomes of oncology patients admitted to the emergency department (ED) with suspected infection.

Methods: Retrospective cohort analysis of all oncology patients presenting to the ED of a tertiary hospital with suspected infection from 1 December 2014 to 1 June 2017. Patients were identified by cross-linkage of ED and Oncology electronic health records. The primary outcome was in-hospital mortality and/or ICU stay $\geq 3$ days.

Results: A total of 1,655 were included in this study - 1,267 (76.6\%) with solid tumour, and $388(23.4 \%)$ with haematological malignancies. At presentation, 495 patients had chemotherapy, and 140 had radiotherapy within the preceding 6 months. 400 patients received chemotherapy and/or radiotherapy in the previous 4 weeks. Overall, 371 (22.4\%) patients had qSOFA $\geq 2$. Such patients had a higher likelihood of respiratory infections compared to patients with a qSOFA $<2(43.9 \%$ versus $29 \%)$ and were more likely to be admitted to ICU or require mechanical ventilation. In-hospital mortality or in-hospital mortality and/or ICL stay $\geq 3$ days were $17.3 \%$ and $21 \%$, for qSOFA $\geq 2$ patients vs $4.7 \%$ and $6.9 \%$ for qSOFA $<2$ patients $(P<0.001)$. qSOFA $\geq 2$ had a negative predictive value $(N P V)$ of $95 \%$ for in-hospital mortality and $93 \%$ for in-hospital mortality or ICU stay $\geq 3$ days.

Conclusion: Among oncology patients presenting to the ED with suspected infection, a qSOFA $\geq 2$ is associated with a 3 -fold risk of hospital mortality/prolonged ICU stay. Its absence helps identify low risk patients.

Key words: cancer, sepsis, septic shock, chemotherapy, radiotherapy, qSOFA 


\section{INTRODUCTION}

Oncology patients are particularly prone to infection (1), and frequently present to emergency departments (ED) with sepsis or septic shock, with as many as 1-in-6 severe sepsis patients in the United States having underlying malignancies (2). A recent Victorian population-based study found an increase in the incidence of sepsis in such patients from $5.8 \%$ in 2008 to $7.8 \%$ in 2015 (3).

To aid the early detection of sepsis, several diagnostic scores have been designed including the Modified Early Warning Score (MEWS), Early Warning Scoring System (EWSS), and National Early Warning Score (NEWS) (4). The Third International Consensus Definitions for Sepsis and Septic Shock (Sepsis-3) has recently redefined sepsis as a "life-threatening organ dysfunction caused by a dysregulated host response to infection", and organ dysfunction as an 'increase in the Sequential (Sepsis-related) Organ Failure Assessment (SOFA) score $\geq 2(5)$.

However, the SOFA score involves various laboratory parameters that are not immediately available in the emergency settings (4). Thus, an abbreviated version with simple key variables was developed based on altered mentation, a respiratory rate $>22 /$ minute, and a systolic blood pressure $<100 \mathrm{mmHg}$ - termed the quick SOFA (qSOFA) (6). The aim of using qSOFA was to achieve rapid risk stratification in patients with suspected infection and identify those with a higher risk of hospital death or prolonged ICU admission $(4,6,7)$. A qSOFA score $\geq 2$ has been assessed as a predictor of in-hospital mortality and prolonged ICU stay among ED patients with suspected infection (6) and reported to have fair sensitivity $(60 \%)$ and good specificity $(80 \%)(8)$.

We hypothesize that qSOFA would also help identify ED patients with cancer and suspected infection at risk of poor outcomes. Accordingly, we aimed to evaluate the utility of qSOFA score in predicting adverse outcomes in patients with cancer who presented to ED with suspected infection. 


\section{METHODS}

\section{Study setting and population}

We performed a retrospective cohort study using the Austin Health electronic health records (EHR) (CERNER Database, Kansas City, MO). All adult patients (aged $\geq 18$ years) who presented to the hospital ED between 1 December 2014 and 1 June 2017 were screened for suspected infection. A suspected infection was defined according to the Third International Consensus Definitions for Sepsis and Septic Shock (Sepsis-3), as the combination of antibiotics and body fluid cultures within a specific time window (9). If the antibiotic was given first, the culture sampling had to be obtained within 24 hours. If the culture sampling was done first, the antibiotic had to be ordered within 72 hours (9). The onset of infection was defined as the time at which the first of these two events occurred. The dataset was crosslinked with the hospital oncology database to identify a subset of cancer patients, which formed the final study cohort (Figure-1). A patient with cancer was defined as any patient who was newly-diagnosed for cancer, had cancer treated by radiotherapy, and/or oral or intravenous chemotherapy, a cytotoxic agent, immunotherapy, or targeted therapy within the preceding 6 months.

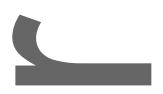

The study was approved by the institutional human research ethics committee with a waiver for informed consent. The STROBE recommendations for the reporting of observational studies were applied to this study (10).

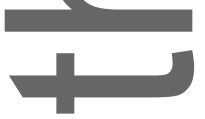

\section{Extracted variables and outcomes definition}

The following key variables were obtained from the EHR: patients demographic features (age and sex), date and time of ED presentation and vital signs values in ED, date of admission and discharge from intensive care unit (ICU) and hospital, date, time and types of body fluid cultures collection (blood, urine, cerebrospinal fluid, etc.) and antibiotic orders, site of infection, and use of life-sustaining treatments (i.e. mechanical ventilation, dialysis, and 
hemofiltration). The oncological data included primary cancer type, and date of last radiotherapy or chemotherapy. The outcomes of interest were in-hospital mortality and ICU stay of $\geq 3$ days.

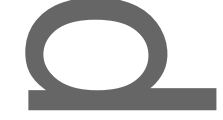

qSOFA score

The qSOFA was calculated using the previously described methodology (6). For all patients, the 3 variables of the qSOFA (highest respiratory rate, lowest systolic blood pressure, and lowest GCS score) were recorded using the worst level for each variable within 3 hours before and after the onset of infection. One point was attributed for the following results: any GCS score <15, any systolic blood pressure $\leq 100 \mathrm{mmHg}$, and any respiratory rate of $\geq$ $22 /$ min. Thus, a final qSOFA score ranged between 0 and 3 . Missing variables were recorded as missing, but assumed to be normal in analyses.

\section{Statistical Analyses}

Descriptive statistics were used to describe the study population. Differences in characteristics and process of care between patients who had qSOFA $\geq 2$ vs. qSOFA $<2$ were comparedusing Wilcoxon rank sum test or Fisher's exact test as appropriate. To assess the performance of the qSOFA to predict adverse events of interest (i.e. in-hospital mortality and ICU stay of $\geq 3$ days), the prognostic performances (sensitivity, specificity, and negative and positive predictive values) of $q S O F A \geq 2$ for each adverse outcomes of interest were calculated. All tests were two-sided and P-values lower than $5 \%$ were considered to indicate significance. Statistical tests were conducted using the SAS 5.0.1 software package (SAS Institute Inc., Cary, CA, USA).

RESULTS

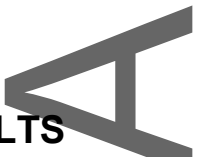

\section{Patient characteristics}


A total of 1,655 oncology patients with suspected infections who presented to ED were included in this study (Figure-1). Baseline characteristics of the study cohort are reported in Table-1. Patients were mostly elderly, with a predominance of males. The majority had solid tumours. Respiratory infections accounted for approximately a third of all suspected infections. More patients had received chemotherapy presentation than radiotherapy in the 6 months preceding ED presentation. The time form last chemotherapy treatment was approximately 10 days and time from last radiotherapy treatment was approximately 2 months.

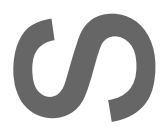

Overall, $371(22.4 \%)$ patients had a qSOFA $\geq 2$ at the time of ED presentation. Patients who had a $q S O F A \geq 2$ were similar in age to those with a qSOFA $<2$. The overall proportion of patients who had $q S O F A \geq 2$ was also similar with regard to solid tumours and haematological malignancies. However, patients with $q S O F A \geq 2$ were less likely to have received chemotherapy in the last 6 months but more likely to have received radiotherapy and more likely to have limitations of medical therapy orders. As expected, patients with qSOFA $\geq 2$ had a lower median blood pressure, higher respiratory rate, and lower Glasgow Coma Scale score.

\section{Process of care}

Process of care-related variables are presented in Table 2. Patients with qSOFA $\geq 2$ were more likely to fulfil the diagnostic criteria for suspected infection earlier and more likely to have blood cultures taken. Their time to antibiotic initiation was shorter and they were more likely to receive cephalosporins and aminoglycosides. Finally, mechanical ventilation was 2.5-fold more common.

\section{Study outcomes}


Patient outcomes are presented in Table-2. More patients with qSOFA $\geq 2$ were admitted to ICU. The percentage of patients with qSOFA $\geq 2$ who died in hospital was approximately 3fold that of patients with qSOFA $<2$. Patients who had $q S O F A \geq 2$ were greater than 3 -times more likely to haye an ICU stay $\geq 3$ days or to die compared to patients who had qSOFA $<2$.

\section{qSOFA as a predictor of adverse outcomes}

For prediction of in-hospital mortality as well as in-hospital mortality or ICU stay $\geq 3$ days, qSOFA $\geq 2$ had greater specificity than sensitivity and thus a strong negative prediction value (NPV) but a low positive prediction value (PPV) (Table-3, Figure-2). Its sensitivity, however, appeared to be greater in oncology patients than in haematology patients, with better areas under the receiving operating characteristic curve. The PPV, NPV, sensitivity and specificity of $q S O F A \geq 2$ in predicting adverse outcomes were similar in sub-cohorts of oncology patients who underwent cancer treatments within 3 months ( $n=523)$ (eTable-1), and 4 weeks of ED presentation ( $n=400)($ eTable-2). In addition, when comparing survivors with non-survivors (eTable-3) - more than $50 \%$ of patients who died were qSOFA $\geq 2$, compared with only $20 \%$ of survivors. On multivariable logistic regression, qSOFA $\geq 2$ was a stronger predictor of mortality than the type of tumour, the recent use of radiotherapy, age and site of infection (eTable-4)

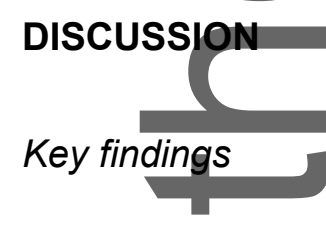

We conducted a study of oncology patients who presented to the ED of a tertiary hospital with suspected infection. We aimed to determine whether a qSOFA $\geq 2$ would help in rapidly identifying those patients at a higher risk of mortality or prolonged ICU admission or both. We found that overall mortality in oncology patients with a qSOFA $<2$ was approximately $5 \%$. We also found that such mortality rate increased approximately three-fold in patients with qSOFA $\geq 2$. Moreover, $q S O F A \geq 2$ had greater specificity than sensitivity and thus a strong 
negative prediction value (NPV). In addition, on multivariable logistic regression, a qSOFA $\geq 2$ was a stronger predictor of mortality than the type of tumour, the recent use of radiotherapy, age and site of infection.

Relationship to previous ED studies

The overall mortality of $7.5 \%$ in oncology patients, irrespective of qSOFA score, was almost twice that previously reported in non-oncology patients presenting to the ED with suspected infection (8). This is consistent with the fact that, in immunocompromised patients, progression of sepsis can be particularly rapid (9). Another possible contributing factor to the increased mortality in oncology patients is less-aggressive intervention due to the presence of treatment limitations. However, in our cohort, there was no significant difference in the percentage of patients with limitations of medical therapy among patients who did or did not die in hospita

In our study, oncology patients with qSOFA $\geq 2$ had an in-hospital mortality rate of $17.3 \%$. Other studies have also reported a significantly higher mortality for patients with qSOFA $\geq 2$ compared to qSOFA $<2$. Freund et al reported a $24 \%$ in-hospital mortality in patients with qSOFA $\geq 2$ versus $3 \%$ in patients with qSOFA $<2$ (hazard ratio for death for qSOFA $\geq 2$ of 6.2 ) (11).

We found that gSOFA's sensitivity for predicting in-hospital mortality was slightly decreased in oncology patients compared to the general ED population in our institution (52\% vs. $61 \%)$. However, there were no major differences in prognostic accuracy between both groups (8). Earlier studies reporting on the prognostic accuracy of qSOFA in non-oncology patients have reported an area under the receiver operating curve (12) which is comparable to ours for both in-hospital mortality, and in-hospital mortality/prolonged ICU admission (12). In a separate single institutional study in the Switzerland, Tusgul et al reported that qSOFA has a sensitivity of $36.3 \%$ in predicting ICU admission, $17.4 \%$ in ICU stay $\geq 3$ days, and $68 \%$ for death within 48 hours (13). In a separate Spanish study involving 1,071 patients who 
presented to ED with suspected infection, qSOFA $\geq 2$ had a sensitivity of $28 \%$ in predicting mortality (14). The study by Freund et al reported a sensitivity of $70 \%$ for $q S O F A \geq 2$ in predicting in-hospital mortality (11).

Study implications

Our findings imply that at the time of presenting to ED with suspected infection, a significant proportion of oncology patients had qSOFA scores $\geq 2$, and that such patients can be rapidly identified as being at much greater risk of mortality and/ or prolonged ICU admission. Finally, they imply that the absence of qSOFA $<2$ can be used to triage patients at low-risk of mortality. It is important to note that oncology patients can be acutely unwell, and their variable prognosis complicates decisions surrounding escalation of care - qSOFA can potentially be utilised to guide complex care escalation processes in several settings e.g. decisions whether to offer ICU beds to oncology patients, or whether to transfer unwell oncology patients from regional hospitals without ICU support to larger tertiary hospitals with

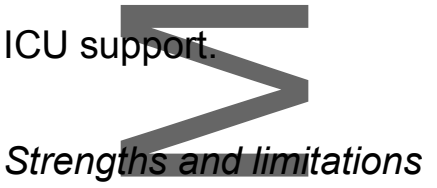

To the best of our knowledge, this is the first and largest observational study of the qSOFA score in predicting in-hospital mortality and prolonged ICU admission in a general oncology population who presented to ED with suspected infection. It provides useful information on the value of $q S O F A$ in the ED for such patients. It found that a $q S O F A \geq 2$ was strong predictor of mortality with high NPV, and that its absence indicates a lower risk state in such patients. Given the ease with which the qSOFA score can be obtained in the ED, our findings suggest that it could be used to help with risk stratification before any laboratory tests become available.

Our study bears the inherent limitations and biases of many retrospective studies. Although we intended to capture a general oncology population, surgical oncology patients who have had surgery but were yet to undergo radiotherapy and chemotherapy were not included in 
this study population. Oncology patients with suspected infections who were direct ward admissions were also not included in this study. Information on other factors that might have influenced mortality were not available such as active cancer vs. remission, and performance status. Lastly, oncology patients are a heterogenous group, with varying physiology and effects from chemotherapeutic drugs. In such a group, there is a chance that any strong associations, or findings that apply to a specific subgroup of patients could have been confounded by others where the association was particularly weak.

\section{CONCLUSION}

Our findings suggest that in a population of oncology patients who presented to ED with suspected infection, qSOFA $\geq 2$ was associated with three-fold increase in hospital mortality or prolonged ICU admission. Moreover, its absence helped identify patients with low risk of such adverse outcomes. Given the ease of measurement of qSOFA variable over just a few minutes and without any need for laboratory tests, our findings support its utility in risk stratification in this setting.

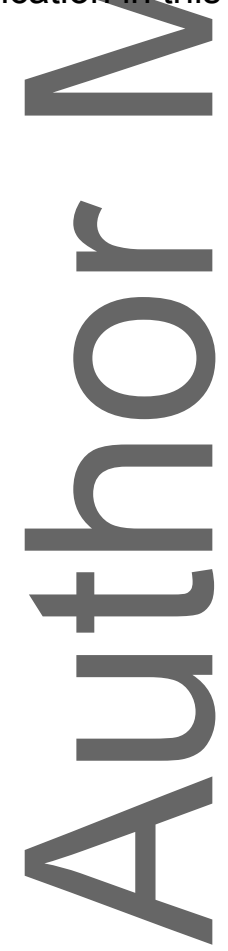




\section{REFERENCE}

1. Rolston KY. Infections in Cancer Patients with Solid Tumors: A Review. Infect Dis Ther. 2017:6(1):69-83.

2. Angus DC, Linde-Zwirble WT, Lidicker J, Clermont G, Carcillo J, Pinsky MR.

Epidemiology of severe sepsis in the United States: analysis of incidence, outcome, and associated costs of care. Crit Care Med. 2001;29(7):1303-10.

3. Te Marvelde L, Whitfield A, Shepheard J, Read C, Milne RL, Whitfield K. Epidemiology of sepsis in cancer patients in Victoria, Australia: a population-based study using linked data. Aust N Z J Public Health. 2019.

4. McLymont N, Glover GW. Scoring systems for the characterization of sepsis and associated outcomes. Ann Transl Med. 2016;4(24):527.

5. Singer M, Deutschman CS, Seymour CW, Shankar-Hari M, Annane D, Bauer M, et al. The Third International Consensus Definitions for Sepsis and Septic Shock (Sepsis-3). JAMA. 2016;315(8):801-10.

6. Seymour CW, Liu VX, Iwashyna TJ, Brunkhorst FM, Rea TD, Scherag A, et al. Assessment of Clinical Criteria for Sepsis: For the Third International Consensus Definitions for Sepsis and Septic Shock (Sepsis-3). JAMA : the journal of the American Medical Association. 2016;315(8):762-74.

7. Vincent JL, Martin GS, Levy MM. qSOFA does not replace SIRS in the definition of sepsis. Crit Care. 2016;20(1):210.

8. Canet E, Faylor DM, Khor R, Krishnan V, Bellomo R. qSOFA as predictor of mortality and prolonged ICU admission in Emergency Department patients with suspected infection. $J$ Crit Care. 2018;48:118-23.

9. Rivers E, Nguyen B, Havstad S, Ressler J, Muzzin A, Knoblich B, et al. Early goaldirected therapy in the treatment of severe sepsis and septic shock. The New England journal of medicine. 2001;345(19):1368-77. 
10. von Elm E, Altman DG, Egger M, Pocock SJ, Gotzsche PC, Vandenbroucke JP, et al. The Strengthening the Reporting of Observational Studies in Epidemiology (STROBE) statement: guidelines for reporting observational studies. PLoS Med. 2007;4(10):e296.

11. Freund Y, Lemachatti N, Krastinova E, Van Laer M, Claessens YE, Avondo A, et al. Prognostic Accuracy of Sepsis-3 Criteria for In-Hospital Mortality Among Patients With Suspected Infection Presenting to the Emergency Department. JAMA. 2017;317(3):301-8. 12. Mellhammar L, Linder A, Tverring J, Christensson B, Boyd JH, Sendi P, et al. NEWS2 is Superior to qSOFA in Detecting Sepsis with Organ Dysfunction in the Emergency Department. J Clin Med. 2019;8(8).

13. Tusgul S, Carron PN, Yersin B, Calandra T, Dami F. Low sensitivity of qSOFA, SIRS criteria and sepsis definition to identify infected patients at risk of complication in the prehospital setting and at the emergency department triage. Scand J Trauma Resusc Emerg Med. 2017:25(1):108.

14. Gonzalez Del Castillo J, Julian-Jimenez A, Gonzalez-Martinez F, AlvarezManzanares J, Pinera P, Navarro-Bustos C, et al. Prognostic accuracy of SIRS criteria, qSOFA score and GYM score for 30-day-mortality in older non-severely dependent infected patients attended in the emergency department. Eur J Clin Microbiol Infect Dis. $2017 ; 36(12): 2361-9$.

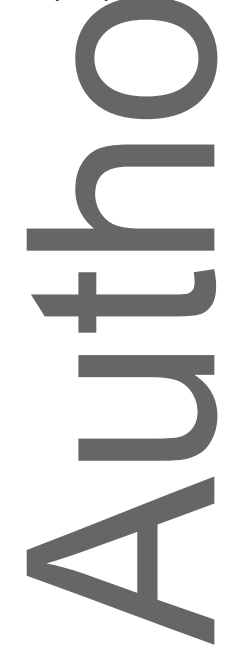


Table 1: Baseline characteristics and process of care of the study cohort

\begin{tabular}{|c|c|c|c|c|}
\hline Variable & $\begin{array}{c}\text { All cancer } \\
\text { patients } \\
\text { with suspected } \\
\text { infection in the } \\
\text { ED } \\
N=1,655\end{array}$ & $\begin{array}{l}\text { Patients with } \\
\begin{array}{c}\text { qSOFA } \geq 2 \\
N=371 \\
(22 \%)\end{array}\end{array}$ & $\begin{array}{c}\text { Patients with } \\
\begin{array}{c}\text { qSOFA<2 } \\
N=1,284 \\
(78 \%)\end{array}\end{array}$ & P-value \\
\hline Age, mean (SD), years & $68.1(15.1)$ & $70.9(14)$ & $67.3(15.4)$ & $<0.0001$ \\
\hline Male gender, $\mathrm{n}(\%)$ & $972(58.7)$ & $236(63.6)$ & $736(57.3)$ & 0.03 \\
\hline Solid tumor, $\mathrm{n}(\%)$ & $1267(76.5)$ & $285(76.8)$ & $982(76.5)$ & \\
\hline Lung cancer & $204(18.6)$ & $53(20.9)$ & $151(18)$ & 0.33 \\
\hline Breast cancer & $199(18.2)$ & $38(15)$ & $161(19.2)$ & \\
\hline Prostat & $151(13.8)$ & $33(13)$ & $118(14)$ & \\
\hline Colorectal cancer & $86(7.9)$ & $18(7.1)$ & $68(8.1)$ & \\
\hline Other & $454(41.5)$ & $112(44.1)$ & $342(40.7)$ & \\
\hline $\begin{array}{l}\text { Haematological malignancy, } \\
\text { n (\%) }\end{array}$ & $388(23.5)$ & $86(23.2)$ & $302(23.5)$ & \\
\hline Non-Hodgkin Lymphoma & $130(46.6)$ & $29(44)$ & $101(47.4)$ & 0.16 \\
\hline Myelom & $45(16.1)$ & $12(18.2)$ & $33(15.5)$ & \\
\hline Acute Myeloid Leukemia & $35(12.5)$ & $8(12.1)$ & $27(12.7)$ & \\
\hline Hodgkin Lymphoma & $24(8.6)$ & $11(16.7)$ & $13(6.1)$ & \\
\hline Other & $45(16.1)$ & $6(9)$ & $39(18.3)$ & \\
\hline $\begin{array}{l}\text { Chemotherapy within } 6 \\
\text { months before ED admission, } \\
\mathrm{n}(\%)\end{array}$ & $495(29.9)$ & $83(22.4)$ & $412(32.1)$ & 0.0003 \\
\hline Time from last chemotherapy & $9.3[4.1-21.2]$ & $11[4.4-36.4]$ & 9.2 [4-19] & 0.09 \\
\hline
\end{tabular}




\begin{tabular}{|c|c|c|c|c|}
\hline $\begin{array}{l}\text { to ED admission, median } \\
\text { [IQR], days }\end{array}$ & & & & \\
\hline $\begin{array}{l}\text { Radiotherapy within } 6 \\
\text { months before ED admission, } \\
\mathrm{n}(\%)\end{array}$ & $140(8.5)$ & $46(12.4)$ & $95(7.4)$ & 0.001 \\
\hline $\begin{array}{l}\text { Time from last radiotherapy } \\
\text { to ED admission median }\end{array}$ & $57.6[37-107.5]$ & $54.2[35.8-78.1]$ & $\begin{array}{c}63.8[37.5- \\
113.5]\end{array}$ & 0.31 \\
\hline qSOFA parameters & & & & \\
\hline $\begin{array}{l}\text { Systolic blood pressure, } \\
\text { mean (SD), } \mathrm{mmHg}\end{array}$ & $112(20.5)$ & $97.9(20.3)$ & $117(18.5)$ & $<0.0001$ \\
\hline $\begin{array}{l}\text { Respiratory rate, mean } \\
\text { (SD), breaths/min }\end{array}$ & $22.4(6.1)$ & $27(6.3)$ & $21(5.2)$ & $<0.0001$ \\
\hline Glasgow Coma Scale, & $14.6(1.1)$ & $14(1.5)$ & $14.8(0.8)$ & $<0.0001$ \\
\hline Site of infection ${ }^{a}, \mathrm{n}(\%)$ & & & & $<0.0001$ \\
\hline Respiratory & $536(32.4)$ & $163(43.9)$ & $373(29)$ & \\
\hline Urinary & $256(15.5)$ & 59 (15.9) & $197(15.3)$ & \\
\hline Abdom & $218(13.2)$ & $43(11.6)$ & $175(13.6)$ & \\
\hline Cutaneous & $100(6)$ & $6(1.6)$ & $94(7.3)$ & \\
\hline Neurological & $7(0.4)$ & $2(0.5)$ & $5(0.4)$ & \\
\hline Other & $12(0.7)$ & $1(0.3)$ & $11(0.9)$ & \\
\hline $\begin{array}{l}\text { Time from ED admission to } \\
\text { onset of infection }{ }^{b} \text {, median }\end{array}$ & $1[0.5-2.1]$ & 0.8 [0.5-1.9] & $1[0.5-2.2]$ & 0.04 \\
\hline $\begin{array}{l}\text { Time from ED admission to } \\
\text { culture order, median [IQR], }\end{array}$ & $1[0.5-2.2]$ & 0.9 [0.5-2] & $1[0.5-2.2]$ & 0.09 \\
\hline
\end{tabular}

This article is protected by copyright. All rights reserved. 


\begin{tabular}{|c|c|c|c|c|}
\hline \multicolumn{5}{|l|}{ hours } \\
\hline Culture tests, n (\%) & & & & \multirow[t]{7}{*}{0.008} \\
\hline Blood culture & $1,140(68.9)$ & $286(77.1)$ & $854(66.5)$ & \\
\hline Urine culture & $378(22.8)$ & $68(18.3)$ & $310(24.1)$ & \\
\hline Respiratory culture ${ }^{c}$ & $87(5.3)$ & $12(3.2)$ & $75(5.8)$ & \\
\hline Wound culture & $35(2.1)$ & $1(0.3)$ & $34(2.6)$ & \\
\hline ospinal Fluid & $3(0.2)$ & $1(0.3)$ & $2(0.2)$ & \\
\hline y fluid & $12(0.7)$ & $3(0.8)$ & $9(0.7)$ & \\
\hline $\begin{array}{l}\text { Time from ED admission to } \\
\text { antibiotic administration, } \\
\text { median [IQR], hours }\end{array}$ & $3.3[2.1-5.3]$ & $2.8[1.7-4.5]$ & 3.5 [2.3-5.5] & $<0.0001$ \\
\hline \multirow{2}{*}{ Antibiotics, $n(\%)$} & & & & \multirow[t]{8}{*}{0.0003} \\
\hline & $807(48.8)$ & $156(42)$ & $651(50.7)$ & \\
\hline orin & $571(34.5)$ & 409 (31.9) & $162(43.7)$ & \\
\hline coside & $56(3.4)$ & $46(3.6)$ & $10(2.7)$ & \\
\hline Tetracycline & $51(3.1)$ & $43(3.3)$ & $8(2.2)$ & \\
\hline Trimethoprim & $35(2.1)$ & $32(2.5)$ & $3(0.8)$ & \\
\hline Mac & $31(1.9)$ & $17(1.3)$ & $14(3.8)$ & \\
\hline Oth & $104(6.3)$ & $19(5.1)$ & $85(6.6)$ & \\
\hline \multicolumn{5}{|l|}{ Life Sustaining Therapies } \\
\hline $\begin{array}{l}\text { Mechanical } \\
\text { Ventilation }\end{array}$ & $142(8.6)$ & $61(16.4)$ & $81(6.3)$ & $<0.0001$ \\
\hline Dialysis & $162(9.8)$ & $43(11.6)$ & $119(9.3)$ & 0.18 \\
\hline Hemofiltrat & $5(0.3)$ & $2(0.5)$ & $3(0.2)$ & 0.31 \\
\hline
\end{tabular}

ED: Emergency Department; IQR: InterQuartile Range; SD: Standard Deviation

${ }^{a}$ Assessed in $1,029(62.2 \%)$ of the 1,655 patients, including $274(73.9 \%)$ of 371 patients with a qSOFA $\geq 2$ and $855(66.6 \%)$ of the 1,284 patients with a qSOFA $<2$. 
${ }^{\mathrm{b}}$ Defined as time of culture or antibiotics order

${ }^{c}$ Including sputum culture $n=83$, throat culture $n=2$, and culture from the respiratory tract $\mathrm{n}=2$.

d Including: Quinolone $n=26$, Nitroimidazole n=25, Glycopeptide n=23, Carbapenem n=16, Lincosamide $n=8$, Rifamycin $n=5$, Oxazolidinone $n=1$.

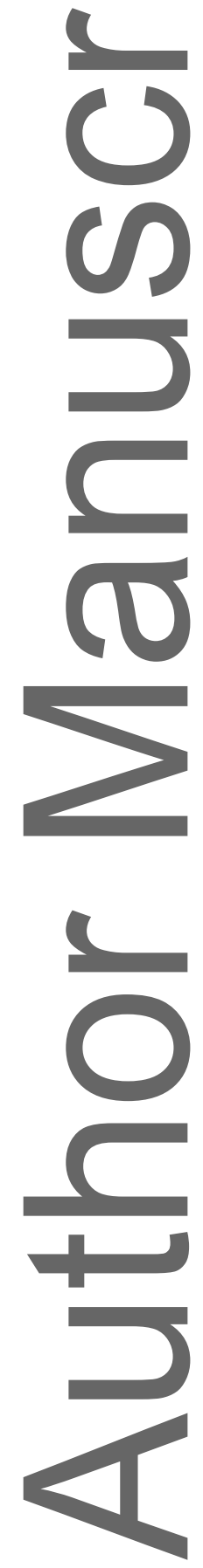


Table 2: Outcomes of interest

\begin{tabular}{|c|c|c|c|c|}
\hline Variable & $\begin{array}{l}\text { All cancer patients } \\
\text { with suspected } \\
\text { infection in the ED } \\
\qquad N=1,655\end{array}$ & $\begin{array}{l}\text { Patients with } \\
\begin{array}{l}\text { qSOFA } \geq 2 \\
N=371\end{array}\end{array}$ & $\begin{array}{l}\text { Patients with } \\
\begin{array}{c}\text { qSOFA<2 } \\
N=1,284\end{array}\end{array}$ & $\begin{array}{c}\text { P- } \\
\text { value }\end{array}$ \\
\hline ED LOS, mean (SD), hours & $6(3.3)$ & $7(3.1)$ & $6.7(3.4)$ & 0.08 \\
\hline Intensive care unit (ICU) & & & & \\
\hline Admission, $\mathrm{n}(\%)$ & $143(8.6)$ & $62(16.7)$ & $81(6.3)$ & $<0.001$ \\
\hline $\begin{array}{l}\text { LOS, median [IQR], } \\
\text { hours }\end{array}$ & 52 [27-98] & $48[27.5-92.5]$ & 55 [27-100] & 0.69 \\
\hline ICU stay $\geq 3$ days, n (\%) & $54(3.3)$ & $20(5.4)$ & $34(2.6)$ & 0.23 \\
\hline ICU mortality, n (\%) & $22(15.4)$ & $12(19.4)$ & $10(12.3)$ & 0.35 \\
\hline $\begin{array}{l}\text { Hospital LOS, median } \\
{[I Q R], \text { hours }}\end{array}$ & 113 [66-211] & $\begin{array}{c}132[78.5- \\
230.5]\end{array}$ & 107 [63-207] & 0.08 \\
\hline In-hospital death, n (\%) & $124(7.5)$ & $64(17.3)$ & $60(4.7)$ & $<0.001$ \\
\hline $\begin{array}{l}\text { In-hospital death or stay in } \\
\text { ICU } \geq 3 \text { days }^{\mathrm{a}}, \mathrm{n}(\%)\end{array}$ & $167(10.1)$ & $78(21)$ & $89(6.9)$ & $<0.001$ \\
\hline
\end{tabular}

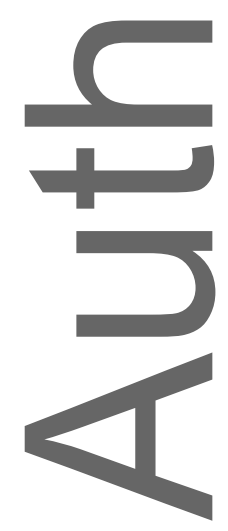


Table 3: Diagnostic performances of qSOFA $\geq 2$ for the prediction of outcomes in oncology patients admitted to the ED with suspected infection.

\begin{tabular}{|c|c|c|c|}
\hline & $\begin{array}{l}\text { Overall cohort } \\
\qquad(n=1,655)\end{array}$ & $\begin{array}{l}\text { Oncology } \\
\text { patients } \\
(n=1267)\end{array}$ & $\begin{array}{l}\text { Haematology } \\
\text { patients } \\
(n=388)\end{array}$ \\
\hline \multicolumn{4}{|l|}{ Hospital mortality } \\
\hline Sensitivity $[\%(95 \% \mathrm{CI})]$ & $52(43-60)$ & $56(46-65)$ & $36(17-55)$ \\
\hline Specificity [\%(95\% CI)] & $80(78-82)$ & $80(78-83)$ & $79(75-83)$ \\
\hline AUROC [\%(95\% Cl)] & $0.70(0.65-0.75)$ & $0.72(0.67-0.77)$ & $0.61(0.51-0.72)$ \\
\hline PPV [\%(95\% & $17(14-21)$ & $19(15-34)$ & $11(5-19)$ \\
\hline NPV [\%(95\% Cl)] & $95(94-96)$ & $96(94-97)$ & $95(92-97)$ \\
\hline LHR+ [\%(95\% & $2.57(2.11-3.14)$ & $2.82(2.29-3.48)$ & $1.70(0.97-2.97)$ \\
\hline LHR- [\% & $0.61(0.50-0.73)$ & $0.55(0.44-0.69)$ & $0.81(0.60-1.10)$ \\
\hline \multicolumn{4}{|c|}{$\begin{array}{l}\text { Hospital mortality or stay in } \\
\text { ICU } \geq 72 \text { hours }\end{array}$} \\
\hline Sensitivity [\%(95\% CI)] & $47(39-54)$ & $51(42-59)$ & $33(19-48)$ \\
\hline Specificity $[\%(95 \%$ CI)] & $80(78-82)$ & $81(78-83)$ & $79(75-83)$ \\
\hline AUROC [\%(95\% CI)] & $0.69(0.65-0.73)$ & $0.71(0.66-0.75)$ & $0.63(0.55-0.71)$ \\
\hline PPV [\%(95\% CI)] & $21(17-26)$ & $23(18-28)$ & $15(8-24)$ \\
\hline NPV $[\%(95 \% \mathrm{Cl})]$ & $93(92-94)$ & $94(92-95)$ & $91(88-94)$ \\
\hline LHR+ [\%(95\% Cl)] & $2.37(1.96-2.87)$ & $2.63(2.14-3.24)$ & $1.59(0.98-2.60)$ \\
\hline LHR- [\%(95\% C)] & $0.66(0.57-0.77)$ & $0.61(0.51-0.73)$ & $0.84(0.67-1.06)$ \\
\hline
\end{tabular}

AUROC: Area Under the Receiver Operating Characteristic curve; $\mathrm{Cl}$, confidence interval; ED: Emergency Department; ICU: Intensive Care Unit; PPV, positive predictive value; NPV, negative predictive value; LHR-, negative likelihood ratio; LHR+, positive likelihood ratio 
Figure-1 | Flow diagram of patients selection for inclusion in the study

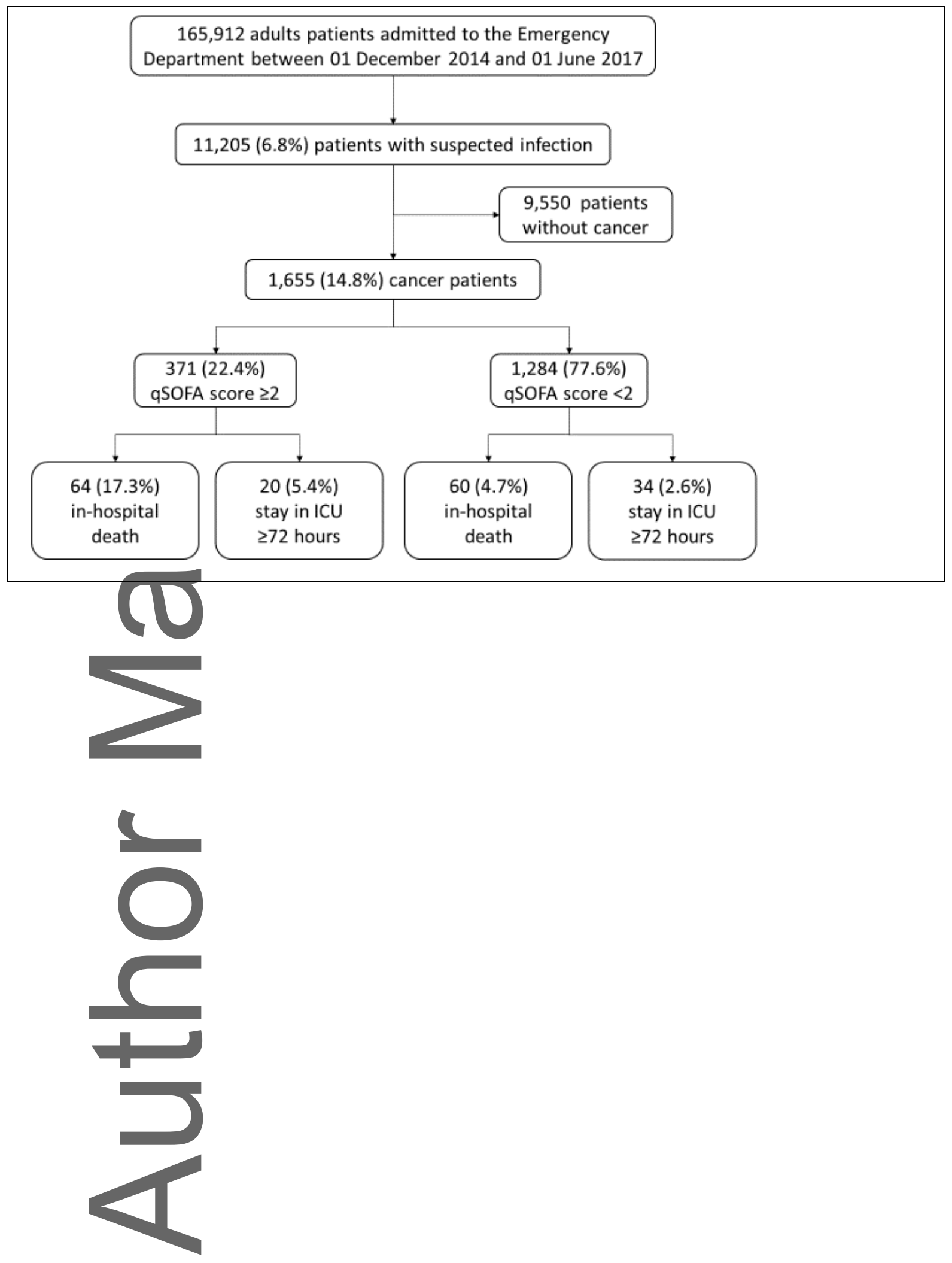

This article is protected by copyright. All rights reserved. 
Figure-2 | Receiving operative characteristic (ROC) curves for A) in-hospital mortality, B) Hospital death and stay in ICU > 72 hours

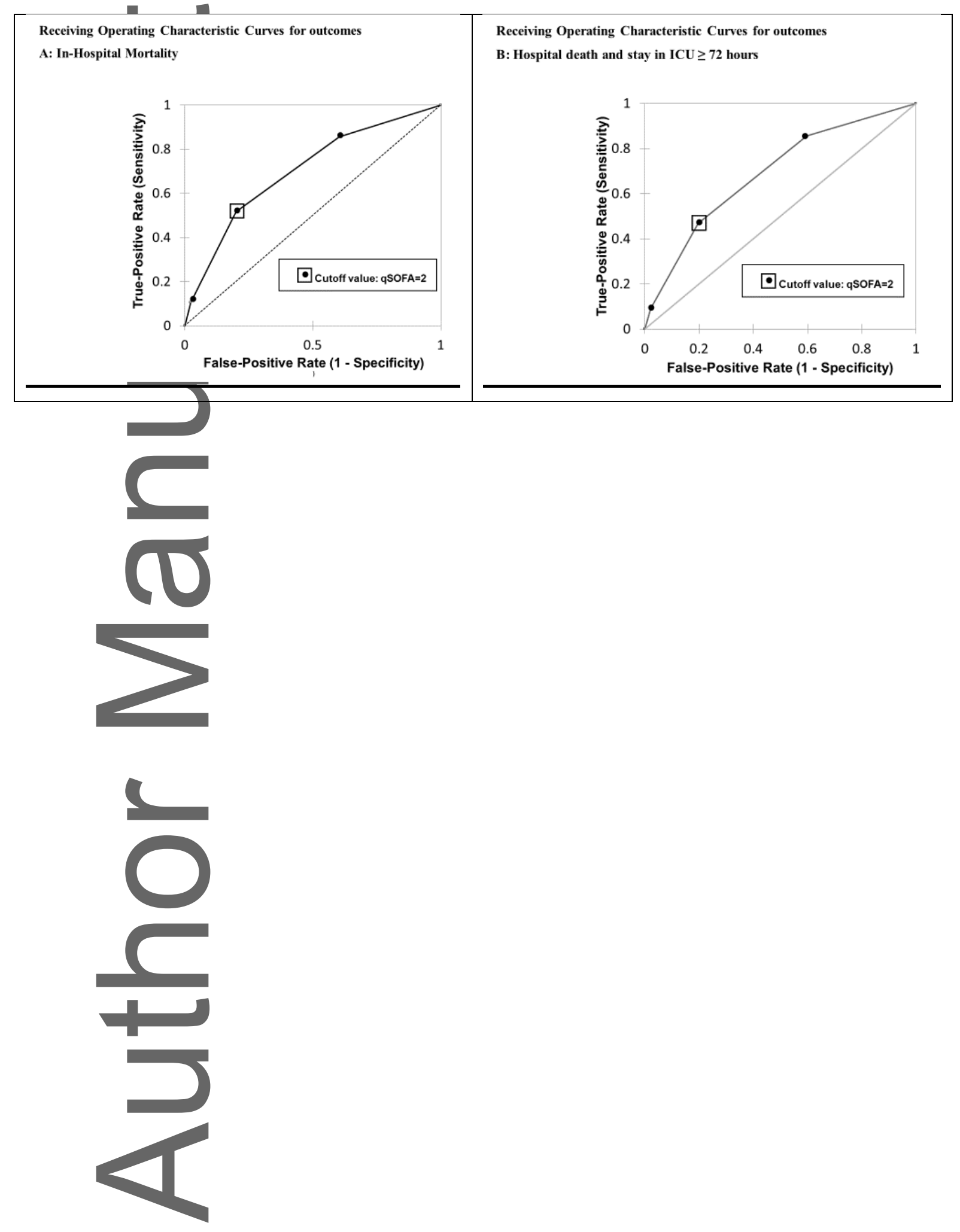

This article is protected by copyright. All rights reserved. 


\section{University Library}

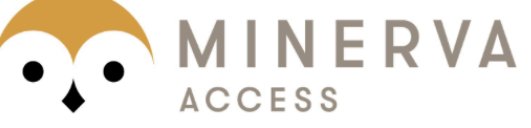

A gateway to Melbourne's research publications

Minerva Access is the Institutional Repository of The University of Melbourne

Author/s:

Koh, TL;Canet, E;Amjad, S;Bellomo, R;Taylor, D;Gan, HK;Marhoon, N;Lim, A;Ong, WL;Krishnan, V;Khor, R

Title:

Prognostic performance of qSOFA in oncology patients admitted to the emergency department with suspected infection

Date:

2021-02

Citation:

Koh, T. L., Canet, E., Amjad, S., Bellomo, R., Taylor, D., Gan, H. K., Marhoon, N., Lim, A., Ong, W. L., Krishnan, V. \& Khor, R. (2021). Prognostic performance of qSOFA in oncology patients admitted to the emergency department with suspected infection. ASIAPACIFIC JOURNAL OF CLINICAL ONCOLOGY, 17 (1), pp.94-100. https://doi.org/10.1111/ ajco.13422.

Persistent Link:

http://hdl.handle.net/11343/276465 